\title{
HEPATITIS B IN A CHILD WITHOUT KNOWN RISK FACTORS
}

Magnolia Cardona, Cissy Chow, Louise McDonnell Western Sector Public Health Unit

O n July 8, 1994 the Western Sector Public Health Unit was notified by a general practitioner (GP) of a case of acute hepatitis $B$ in a child under the age of 12 . The child presented to the GP with the mother on June 6, 1994 with a history of feeling generally unwell and was found to be jaundiced. Blood tests revealed the child had abnormal liver function tests, was positive for hepatitis B surface antigen and had IgM for hepatitis B core antigen. On questioning the GP found there had been no known contact with a case or carrier of hepatitis B virus (HBV). The child had not had surgery, acupuncture, tattoos, blood transfusions, blood products or donor organs. The child had not been exposed to injected drugs or human bites. There was no known sexual contact and the mother believed this was very unlikely. The child had attended a dental surgery for an initial assessment on May 5, 1994 and for a dental filling on June 15.

Both parents and the child were born in Australia. The GP ascertained that members of the child's family were all negative for hepatitis B antigens. The mother completed a course of hepatitis B vaccination in February 1994 and as a result was hepatitis $B$ surface antibody positive. She had no known risk factors for hepatitis $B$ and the reason she was vaccinated is unknown.

Exposure to dental intervention six weeks before the onset date and lack of other risk factors for hepatitis B raised concerns that the child may have acquired the disease at the dental practice. In the interest of the health of the public, and given the GP's concern, the situation provided sufficient grounds to pursue an investigation of the potential sources of infection.

\section{THE INVESTIGATION}

The child had a dental filling five days before the onset of symptoms. However, based on the incubation period reported in the literature (usually $45-180$ days, average $60-90$ days and as short as two weeks) ${ }^{1}$, we ruled out the possibility of transmission at this visit and focused our investigation on the earlier dental consultation. The dental practice employed several dentists and dental assistants.

Using the guidelines for prevention of transmissions of viral infections in dentistry issued by the US Centers for Disease Control and Prevention ${ }^{2}$ and the Australian National Health and Medical Research Council ${ }^{3}$ (NHMRC), we designed a questionnaire and administered it to two dentists and one dental assistant directly involved in the care of the child. The questionnaire aimed to establish potential sources of cross-contamination from the dental staff or other patients attending on the day investigated. It included questions on immunisation status of staff members.

An infectious disease control expert from a dental school assisted in the transcription of dental records of all patients who attended the practice the same day as the child, the day before and the day after. From these records we obtained information on patients' self-reported hepatitis B status and risk factors for the disease, the invasiveness of the procedure performed and whether there was any needlestick accident or parenteral exposure documented.

\section{FINDINGS}

The child and most other patients attending the practice on the day investigated had had a dental examination only that is, a non-invasive procedure where the possibility of blood spilling is negligible unless there is serious gingivitis. No written evidence of needlestick accidents or any other high-risk events was found in any of the records reviewed, including the child's record. There was no history of past hepatitis B infection documented in dental notes of any of the patients attending on the day investigated, the day before or the day after.

From the interviews of dental staff we concluded that the dentists had a reasonable level of awareness of infection control practices but the dental assistant was more knowledgeable on cleaning procedures. This was expected as assistants, unlike dentists in this practice, are directly involved in the disinfection and sterilisation of equipment as well as in the disposal of waste. Overall, the staff's knowledge of management of a needlestick injury was unsatisfactory.

All dentists and the only senior dental assistant allowed to perform procedures without supervision had been immunised against hepatitis B within the past eight years. All had had a booster immunisation after five years or satisfactory antibody levels tested within the past year. Three junior dental assistants had not received any hepatitis B immunisation and one had received two doses. Neither of the junior assistants had direct involvement in patient procedures.

We matched the initials, date of birth, gender and postcode of all dental staff and some 30 patients attending the day of the consultation, the day before and the day after against the NSW hepatitis B notification database (IDSS) at the NSW Health Department. There were no matches found.

We did not conduct a formal inspection of the premises, but staff interviews took place in the premises and this gave us the opportunity to cross-check some of the information provided. The dental consultant was satisfied there was nothing unusual about the practice except for some deficiencies in record-keeping.

\section{DISCUSSION}

Our findings suggest the dental practice was unlikely to be the source of the child's hepatitis B infection because:

- the date of the dental filling was too close to the onset date;

- the earlier consultation did not involve an invasive procedure;

घ there were no matches for hepatitis B in the IDSS database among dental staff or patients undergoing procedures on the same day as the child;

- staff members performing direct patient procedures were immunised; and

- knowledge of infection control among staff was satisfactory. 


\section{Hepatitis B in a child}

\section{Continued from page 123}

We provided copies of the NHMRC guidelines for infection control in dentistry and the Australian National Council on AIDS guidelines on management of needlestick injuries ${ }^{4}$ to the staff and recommended:

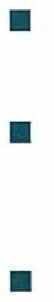

the unimmunised dental assistants be screened for hepatitis B status and, if applicable, be immunised as soon as possible;

the practice implement stricter control of needlestick injuries by keeping a log book and by familiarising staff with the NHMRC guidelines in case of accidents of this kind; and specific inadequacies in record-keeping be corrected in future for the benefit of the practice.

We were unable to determine the source of hepatitis $B$ infection in this case.

Horizontal transmission of HBV (through saliva, wounds) among children aged less than 10 years is common in endemic countries. It is also possible among preadolescents and young school children in developed countries despite low prevalence of carriers in those age groups $s^{5}$. The extent of cross-infection in primary school children in Sydney is thought to be low even in schools with a large concentration of "high-risk" children". In a NSW rural community with a high proportion of Aborigines, markers for HBV were significantly more prevalent among Aborigines than non-Aborigines and many of the seropositive children had been infected before school age, suggesting vertical rather than horizontal transmission?. Our investigation found no other cases of HBV among children had been notified within Western and Wentworth Health Areas since the local IDSS was established in 1991, and there was no history of human bites or wounds so this route was considered unlikely.

This investigation has not been able to exclude the possibility of other routes of exposure to $\mathrm{HBV}$, including sexual exposure.

The potential risk of cross-infection with hepatitis B in dental settings to and from patients has been recognised for some two decades ${ }^{8,9,10}$ and several studies have reported a high prevalence of $\mathrm{HBV}$ carriers among dental professionals ${ }^{11,12,13,14}$. None of the investigated dental staff had been notified to the Health Department as HBV positive and all those directly performing patient procedures had been immunised. We relied on the dental staff's self-report of hepatitis B immunisation and antibody status, lack of personal history of the disease and lack of matches to the IDSS to assess the carrier status of staff. We did not serologically test any of them but think it unlikely any was an asymptomatic carrier of HBV. We recognise that some cases of $\mathrm{HBV}$ occurring before the establishment of IDSS may have gone unnotified. Testing of staff may be appropriate for other specific investigations.

Immunisation against $\mathrm{HBV}$ is considered the frontline protective measure for workers in dentistry ${ }^{11,15}$. Gloves, masks and eyeware alone offer limited protection ${ }^{15}$. Educating health workers on infection control and encouraging the implementation of practice guidelines continue to be basic strategies for the prevention of patient-to-patient and worker-to-patient infections. Dental staff at the practice had a sound knowledge of the universal precautions for prevention of bloodborne infections but less than optimal level of awareness of management guidelines in the event of injuries with sharps.

The NSW Health Department has established a special committee to assess strategies leading to adequate implementation of infection control guidelines in medical and dental settings. The committee will also provide expert advice on the nature and extent of future investigations on infection control.

\section{ACKNOWLEDGMENTS}

Thanks are due to the following people involved in the investigation: the treating GP; staff from the dental practice for their time and co-operation; Rob Menzies for data matching; Ron Robinson for expertise in planning and record review; staff from the Dental Branch and AIDS/Infectious Diseases Branch of the NSW Health Department and the Dental Hospital for their input on the design of the questionnaire; and Louisa Jorm for her input in the draft of this paper.

1. Benenson AS, Editor. Control of communicable diseases in man. An official report of the American Public Health Association. 15th ed, Washington, 1990; 203 .

2. US Centers for Disease Control and Prevention. CDC recommendations for infection control practices. MMWR 1993; (41)8:1-12. 3. NHMRC Dental Health Committee. Guidelines for the prevention of transmission of viral infection in dentistry. Australian Government Publishing Service, Commonwealth of Australia 1992; ISBN 0644289643. 4. ANCA. Management of exposure to blood/body fluids contaminated with blood, including needlestick/sharps injuries, with a potential for HIV or other bloodborne infections. Australian National Council on AIDS, Bulletin No 16

5. Davis LG, Weber DJ, Lemon SM. Horizontal transmission of hepatitis B virus. Lancet 1989; 889-893.

6. Burgess MA, McIntosh EDG, Allars HM, Kenrick KG. Hepatitis B in urban Australian schoolchildren. No evidence of horizontal transmission between high-risk and low-risk groups. Med J Aust 1993; 159:315-319. 7. Camphell DH, Sargent JW, Plant AJ. The prevalence of markers of infection with hepatitis B virus in a mixed-race Australian community. Med J Aust 1989;150:489-492.

8. Levin ML, Maddrey WC, Wands JR, Mendeloff AI. Hepatitis B transmission by dentists. JAMA 1974; 228:1139-1140.

9. Rimland D, Parkin WE, Miller GB, Schrack WD. Hepatitis B outbreak traced to an oral surgeon. New Engl J Med 1977; (296)17:953-958. 10. Alter HJ, Chalmers TC, Freeman BM, Lunceford JL et al. Healthcare workers positive for hepatitis B surface antigen. Are their contacts at risk? New Engl J Med 1975; 292:454-457.

11. Radvan GH, Allworth AM, Hardy GL, Keller AJ. Hepatitis B markers in health care workers. The Newcastle Study. Med J Aust 1984 140: 583-585.

12. Feldman RE, Schiff ER. Hepatitis in dental professionals. Gastroenterology 1973; 65:A-15/539.

13. Cundy KR, Kleger B, Hinks E, Miller LA, editors. Infection control dilemmas and practical solutions. New York: Phenum Press, 1990; 121. 14. Hardt F, Aldershvile J, Dietrichson O, Juhl E et al. Hepatitis B virus infection among Danish surgeons. J Infect Dis 1979; (140)6:972-974. 15. Reingold AL, Kane MA, Hightower AW. Failure of gloves and other protective devices to prevent transmission of hepatitis B virus to oral surgeons. JAMA 1988; 259:2558-2560. 